\title{
Future of Surgery and Shrinking Surgeon
}

\author{
Ajay K. Khanna $\cdot$ Rahul Khanna
}

Published online: 8 February 2011

(C) Association of Surgeons of India 2011

During no other period in the history of mankind have advances occurred in the field of medicine with as much speed and intensity as the last two decades. Many things which are considered routine today were unimaginable barely twenty years ago. There has been a paradigm shift in the treatment of several disease conditions in almost all disciplines of surgery. Such drastic changes have happened because of two prime reasons. One is the technological break through which have occurred in both diagnostics and therapeutics. Second is better understanding of the disease process especially the etiology and the natural course of diseases.

One pertinent question that springs to mind, 'What does the future hold for surgery and surgeons". Have we reached a plateau in the growth spurt of the last 20 years as will the future again redefine the reach and scope of the surgeon. In reference to minimal invasive surgery, several people are already saying 'A surgeon without a scope (endoscope) has no scope (future)'. The indications are already visible to us.

How much this statement is true was answered 120 years ago by Dr. David W. Cheeres, MD, President of the American Surgical Association. In an article published in Boston Medical and Surgical Journal in 1889 he wrote "I believe that we are warranted in saying that the future of surgery is without limit". I deduce this conclusion from considering what the mind of man has already done; second from the future possibilities of field hitherto unknown and unexplored but now opening up to science. There can be but two limitation, either in the mind of man or in the

A. K. Khanna $(\bowtie) \cdot$ R. Khanna

Department of General surgery, Institute of Medical sciences,

Banaras Hindu University,

Varanasi, India

e-mail: akhannabhu@gmail.com subject' [1]. Further he says that the brain has progressed from the rude hammer of the prognathous cave dweller to the telescope and the microscope. Even today we are depending on these technologies. What is there today is the telescopic vision whether by the side of the patient or by a telescopic vision utilized to carry out cholecystectomy sitting 7000 miles away (Lindbergh operation) [2]. Though it is the surgeons mind, but all is possible because of Information and Technology and we must be grateful to our non medical colleagues what we rightly can say as "Bioengineers" to provide us such technology. Who could have thought that a miniaturised surgeon will go inside the gut and will correct the various abnormality without any scar on the body. So the surgery is moving toward a scarless surgery. What ever we do if we put a knife over the skin, it will heal by scarring so only way to give scarless surgery is by what is rightly called as Natural orifice surgery [3]. We are looking at a knife by which we make a incision on the skin but there is no scar which is supposed to be possible only "in utero" surgery but I am sure there will be one day such a knife will be available which will leave no scar [4]. Various types of energy devices are available to make the surgeons life easier and it will be seen that such energy device in future will heal the wound without scarring.

What a great improvement in diagnostic and interventional modalities. Only thirty year back it was so difficult to diagnose gall stone and the surgeon were relying only on cholecystography which many a times gave dubious results but ultrasound and its resolution can make almost the $100 \%$ diagnosis of gall stone and can guide the surgeon for the type of surgery whether it is conventional open surgery or laparoscopic surgery or Natural orifice surgery [3]. Probably we have reached the maximum in term of gall stone treatment by surgical method by miniaturizing the technol- 
ogy but the day will be not far where some medicines will be introduced which will be curing this condition without surgery. What happened in the peptic ulcer surgery which is being treated only by medical treatment now until unless complicated?

The combination of technical advances, miniature vision technology, nanotechnology and robotic technology have opened up new vistas which are yet to find other rightful places in surgical text books. Fundamental conceptual changes especially in the biological behaviour of the cancer cells have made drastic changes in oncological care. Cancer vaccines have become the highlight of the day like in cervical cancer [5].

The role of the bioengineer will become crucial. Almost all advances have been technology driven and our dependence on technical expertise will be even more in the future. The care and maintenance of equipment and their continuous upgradation is beyond the scope of surgeons. It might even happen that the bioengineer will be in the not too distant future become the 'master of the OT' and the role of surgeon would be secondary to him.

The mechanical efficiency of the robotic devices have been shown to be superior to human ergonomics. Transatlantic robotic assisted remote telesurgery have proved it. In the next few years the prices of the operating robotic system is going to come down substantially as manufacturers become free of patent restrictions. This will open up a new dimension by increasing the safety and range of minimal access surgery tremendously. In several western countries and even in metro cities of India the future of open surgery would be in doubt.

As a surgeon we should feel cherished that the medicine only subside the disease but hardly it is near to cure and it is only the surgeon who can think of curing the disease. Diabetes was supposed to be a life long disease and the patient had to be on medication life long but the advent of metabolic / bariatric surgery (apart from transplantation) has really made a real thrust on this disease and curing it within few hours to few days of surgery. This is the concept of 'physiological repair'. The three prime examples of this are metabolic surgery, stem cell therapy and gene therapy. All three in same way or the other correct the underlying etiological factor responsible for that disease condition. Who would have thought diabetes and obesity would be surgically corrected rather than with medicines. Stem cell therapy and gene therapy have such great prophylactic potential that the role of all other surgical and medical specialties would pale in insignificance.

But I think the nature is more smarter than the human race. We invented the wheels, the pendulum, the siphon, under water in the space, in the infinite of microorganism, the mind finds no limit but is ever restless, ever searching but two things still we are not able to conquer, the pain and sepsis. In spite of so many techniques to alleviate the pain but still no technique is full proof. For the sepsis I think we are falling in our own ditch, the microbes are much more smarter than the magic bullets (antibiotics) and it is expected that in the next 20 years there will be no new antibiotic to tackle these small tiny things. Microbes started their groupism and started forming the Biofilm which is almost resistant to current antimicrobials [6]. With the percentage of immuno-compromised population on the rise, sepsis related mortality will continue to challenge us in the next few decades.

The crucial issue for the patient may will be : will there be a doctor to care for me. It is now been suggested that by the year 2020, there will be a large shortage in the physician work force, now more obvious in the discipline of surgery. A survey of 70 medical schools in 35 states in USA reported shortages in surgeons by about $20 \%$. A survey by the Massachusetts Medical Society suggest a physician shortage in general surgery of $32 \%$ with Orthopedics, Neurosurgery and Urology having even greater shortages. The increasing number of elderly and their increasing utilization of the health care and the diminishing popularity of the medicine are the most important. Given a choice between a specialist and a generalist, the patient want to have specialist and it is compounded by readily available information on the net with beautiful coloured pictures showing the supremacy in the delivery of the contents. The patient is on the advantage that he can search as much on the net for his own disease only while surgeon does not a have a time to look into so many conditions which he face so the scenario may be that the patient may be offended when surgeon cannot tell about a recent study published. Increasingly the patients are asking to see the result of intervention before they select their doctors or hospitals. Clinical care is becoming patient centered and outcome based and the patient will have more and more say in their practice of treatment.

As Indian surgeons, still we feel lucky that the patients still think that we are Gods and what ever we do is the best but the time is not far where our patient will be much educated and will sit by the surgeon to explain each and every thing involved in their treatment.

Surgeon is becoming like an Engineer who controls the things with the remote but can we compare the human body with the things. On July 11, 2000 FDA approved the first completely robotic surgery device, the Da vinci surgical system which enable surgeon to remove the gall bladder by sitting on a computer console. Manufacture believes that their products will broaden the scope and increase the effectiveness of the minimal invasive surgery, improve patient outcome and create a safer, more efficient and more effective operating room [7]. 
The industry is overpowering the surgery and surgeon is becoming the doll in the hands of industry [8]. The day may not be far when surgeon may not enter in the operation room, may have no direct touch to the patient, may not have any conversation face to face and that is the "shrinking surgeon".

\section{References}

1. Cheever DW (1889) The future of surgery without limit. Boston Med Surg J 120:501-503
2. Marescaux J (2001) Transatlantic Robotic Assisted Remote Telesurgery. Nature 413:379-80

3. Cahill Ronan A (2010) Natural orifice Tran luminal Endoscopic Surgery-here and now. Surgeon 8:44-50

4. Willyard C (2008) Tinkering in the womb: the future of fetal surgery. Nat Med 14:1176-7

5. Hellner K, Munger K (2011) Human Papilloma Viruses as therapeutic targets in human cancer. Jan 10 (Epub ahead of print)

6. Randal D, Garth DE (2008) Biofilms and chronic infections. JAMA 299:2682-4

7. Graber John N (1990) Open Forum. The future of surgery is "Less invasiveness". J Laparoendo Surg 1:57

8. Peter Bell (2003) What is the future of surgery in the UK. Surgery 21:i-iii 